\title{
Mobile based Automated Complete Blood Count (Auto-CBC) Analysis System from Blood Smeared Image
}

\author{
Cham Ying Kit ${ }^{1}$, Razali Tomari ${ }^{2}$, Wan Nurshazwani Wan Zakaria ${ }^{3}$, Nurmiza Othman ${ }^{4}$, \\ Syadia Nabilah Mohd Safuan ${ }^{5}$, Jacqueline Ang Jie $\mathbf{Y i}^{6}$, Nicholas Tan Chun Sheng ${ }^{7}$ \\ ${ }^{1,2,3,5}$ Department of Mechatronic and Robotic Engineering, Faculty of Electrical and Electronic Engineering, \\ Universiti Tun Hussein Onn Malaysia, Malaysia \\ ${ }^{4,6,7}$ Department of Electronic Engineering, Faculty of Electrical and Electronic Engineering, \\ Universiti Tun Hussein Onn Malaysia, Malaysia
}

\begin{tabular}{l}
\hline \hline Article Info \\
\hline Article history: \\
Received Jun 6, 2017 \\
Revised Sep 2, 2017 \\
Accepted Sep 20, 2017
\end{tabular}

\section{Keyword:}

Colour space analysis Complete blood count (CBC) Contour detection Hough circle transform (HCT)

Red blood cell (RBC)

White blood cell (WBC)

\begin{abstract}
Blood cells diagnosis is becoming essential to ensure a proper treatment can be proposed to a blood related disease patient. In current research trending, automated complete blood count analysis system is required for pathologists or researchers to count the blood cells from the blood smeared images. Hence, a portable mobile-based complete blood count (CBC) analysis framework with the aid of microscope is proposed, and the smartphone camera is mounted to the viewing port of the light microscope by adding a smartphone support. Initially, the blood smeared image is acquired from a light microscope with objective zoom of $100 \mathrm{X}$ magnifications view the eyepiece zoom of $10 \mathrm{X}$ magnification, then captured by the smartphone camera. Next, the areas constitute to the WBC and RBC are extracted using combination of color space analysis, threshold and Otsu procedure. Then, the number of corresponding cells are counted using topological structural analysis, and the cells in clumped region is estimated using Hough Circle Transform (HCT) procedure. After that, the analysis results are saved in the database, and shown in the user interface of the smartphone application. Experimental results show the developed system can gain $92.93 \%$ accuracy for counting the RBC whereas $100 \%$ for counting the WBC.
\end{abstract}

Copyright $\odot 2017$ Institute of Advanced Engineering and Science. All rights reserved.

\section{Corresponding Author:}

Razali Tomari,

Department of Mechatronic and Robotic Engineering,

Faculty of Electrical and Electronic Engineering,

Universiti Tun Hussein Onn Malaysia,

Parit Raja Batu Pahat, Johor, Malaysia.

Email: mdrazali@uthm.edu.my

\section{INTRODUCTION}

Complete blood count (CBC), also known as blood counting [1], is a basic screening test which is performed frequently in laboratories [2]. It counts the quantity of different types of major cells in the blood sample, which are red blood cells (RBC), white blood cells (WBC) and platelets [3]. Besides, CBC provides some crucial information on some parameters that related to each type of blood cells for determining an individual's general health status [4].

For conventional implementation of $\mathrm{CBC}$ analysis, there is a limitation that $\mathrm{CBC}$ analysis is typically accomplished in specific laboratories with trained laboratory techinicians. The laboratory is equipped with high end optical instrument, and manual counting is usually done by a trained laboratory technician or a doctor which causes time wasting while transferring the blood samples to the laboratory and waiting for the results. Indeed, it is a laborious task and may lead to human variation error. 
Instead of implementing the $\mathrm{CBC}$ analysis manually, there are few technologies used to count the blood cells in a specimen automatically, such as image-based cell counters [5], flow cytometers [6] and coulter counters [7]. However, these techniques still require expertise to operate it and the equipment for these methods are expensive. Hence, a more convenient and reliable method to automatically count the blood cells in a specimen is introduced which is a mobile based automated complete blood count analysis system [8]. This creates new opportunities for more efficient medical care system [9]. Besides that, this method also bring benefits to developing country because the cost is lower as compared to other methods [10].

Although an automated blood cell counter is effective in counting blood cells, but it is hard to be implemented in all hospitals and clinics in developing country due to the equipment used is very bulky, costly and need to be operated by trained technicians [11]. Apparently, the maintenance is also costly. Instead of purchasing a new automated blood cell counter machine, it is more practical and portable to develop an automated complete blood count analysis system that is to be implemented by using mobile devices such as smartphones [12]. The smartphone camera lens attached to the microscope is capable to provide digital view of the blood cell and the image processing support can be easily ported to the smartphone application development software.

Hence, we propose a mobile based automated complete blood count analysis system from blood smeared images to count the number of RBC and WBC by considering an overlapping constraint. This paper is organized as follows: Section 2 presents the methods of this proposed project; Section 3 contains the results; Section 4 shows the discussion; and Section 5 is the conclusion of this project.

\section{RESEARCH METHOD}

In this section, a well-planed project methodology is discussed and elaborated. The following Figure 1 show the overall methodology in this project. In Figure 1, the methods are briefly presented and separated into two major phases, which are image acquisition and application building phase. In application building, it is separated into three minor components, which are preparing segmentation and counting algorithm, setting storage and database, and designing user interface (UI).

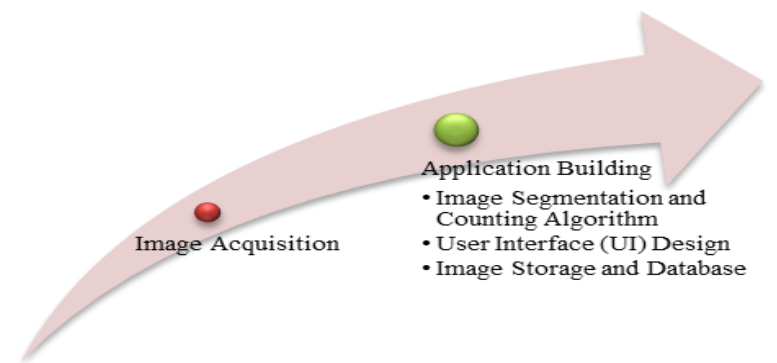

Figure 1. Overall methodology which includes image acquisition and application building

\subsection{Image Acquisition}

First, the image is acquired and captured by Sony 21 Megapixel CMOS image sensor, IMX230, which the camera is attached on the smartphone of Sony Xperia M5. With the aid of light microscope, the blood smeared images are captured in 100X objective magnification and 10X eyepiece magnification. The following Figure 2 shows the setup for the image acquisition.
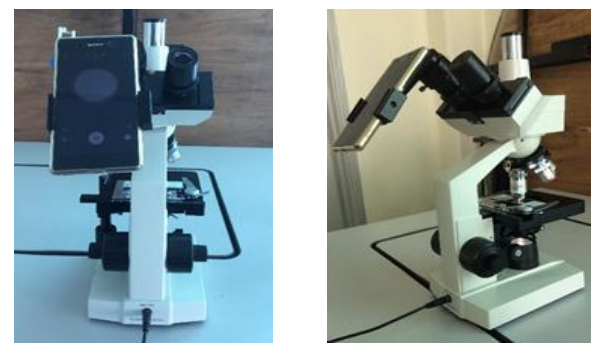

Figure 2. Smartphone attached on the light microscopes 


\subsection{Application Building}

To utilise the smartphone that had been used for acquiring the microscopic images, the application software has been developed by using Android Studio with the API 21 support, which compatible with Android Lollipop operating system (OS).

Hence, there are three components has been developed using Android Studio, which are image segmentation and counting algorithm design, storage and database management, and user interface (UI) design.

\subsubsection{Image Segmentation and Counting Algorithm Design}

In this stage, the blood smeared images are processed to obtain the number of red blood cells and the number of white blood cells. With the aid of OpenCV libraries, which ported as a module in Android Studio project, all the acquired images are able to be processed to obtain useful information, such as the numbers of RBC and WBC from an image. A general process flow of image segmentation and counting is presented in Figure 3.

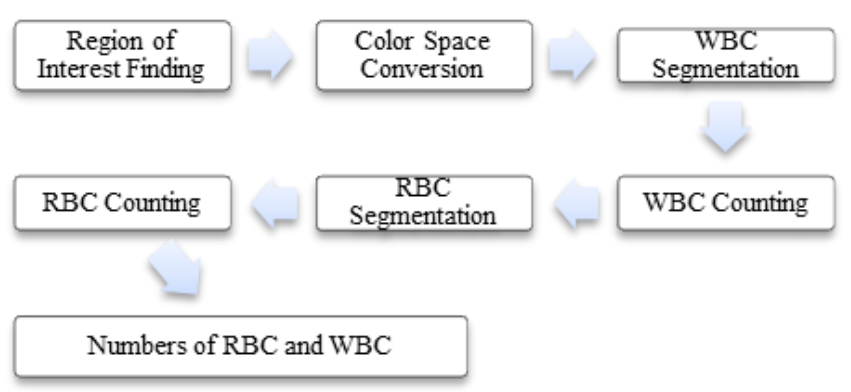

Figure 3. General process flow of image segmentation and counting

The first step is to find the region of interest (ROI) in the images as the image captured is approximately circular in shape. Figure 4 shows the detailed processed on finding the ROI of an image.

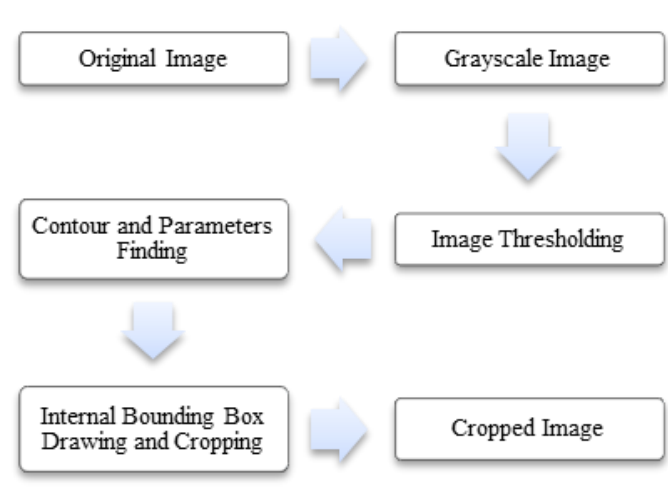

(a)



Original Image

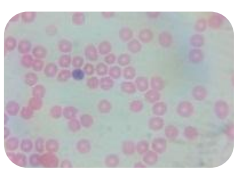

Cropped Image

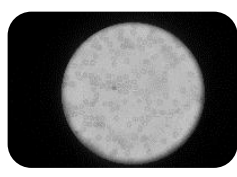

Grayscale Image


Internal Bounding Box
Drawing and Cropping

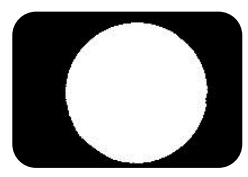

Image Thresholding

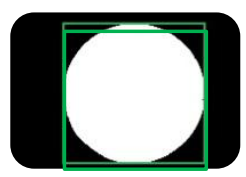

Contour and Parameters
Finding

(b)

Figure 4. Detailed process in finding and cropping the region of interest from images in (a) flow chart and (b) image illustrations for each stage of processing to crop the image

Initially the original image is being grayscale, before it passes through thresholding process. Next, the grayscale image is transformed into binary image using Otsu threshold for differentiating the ROI and background of the images. Then, the binary image is process under contour finding to obtain the information of the ROI. In finding contour information, the height, width, position and centre of the contour are extracted 
and used for drawing internal bounding box and cropping the ROI into a new image. Figure 5(a) illustrates how the internal bounding box to be drawn on a contour.

In Figure 5(a), for drawing internal bounding box (red square in Figure 5(a)), the height and width of the internal bounding box are set to be equal. This is because the diameter of the contour (black oval shape in Figure 5(a)) varies the height and the width of the contour (green rectangle in Figure 5(a)). By determining the precise diameter (orange line in Figure 5(a)) to be used for drawing the internal bounding box, the minimum value is chosen by comparing the value of the height and width of the contour. Then, the value multiplies with $\sin 45^{\circ}$ or 0.7071 as using the trigonometric resolver to determine the width and the height of the internal bounding box.

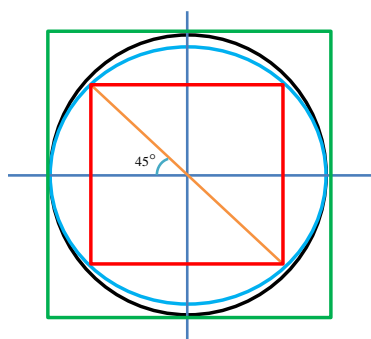

(a)

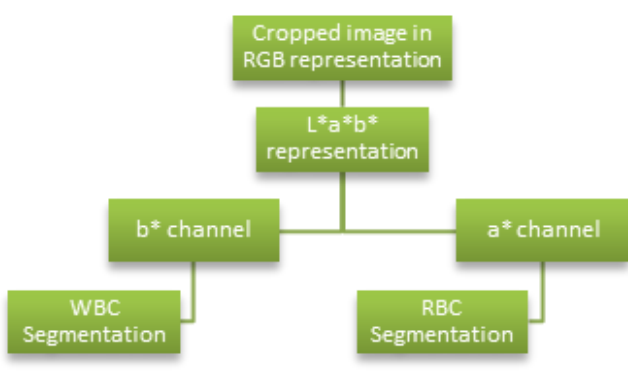

(b)

Figure 5. (a) Illustration of the cropping on internal bounding box (red color box). (b) Cropped image in RGB representation is converted to $\mathrm{L}^{*} \mathrm{a}^{*} \mathrm{~b} *$ colour space. $\mathrm{b}^{*}$ channel is used for WBC segmentation whereas $\mathrm{a}^{*}$ channel is used for RBC segmentation conversion.

Hence, the original image is cropped according to the internal bounding box for colour space

In color space conversion phase, the cropped image is transformed into $\mathrm{L}^{*} \mathrm{a} * \mathrm{~b} *$ representation. Figure 5(b) shows the conversion and separation of the colour space during this phase. The cropped images is converted from RGB colour space to $\mathrm{L}^{*} \mathrm{a} \mathrm{b}^{*}$ colour space [13]. In this project, channel $\mathrm{b}^{*}$ and channel $\mathrm{a}^{*}$ from the $\mathrm{L}^{*} \mathrm{a} \mathrm{b}^{*}$ colour space are used for WBC and RBC segmentation respectively.

In WBC segmentation and counting, the WBC in $b^{*}$ channel image is segmented using binary threshold. Next, WBC is counted based on the number of contours in the segmented image using topological structural analysis [14]. Figure 6 illustrates the process of WBC segmentation and counting.
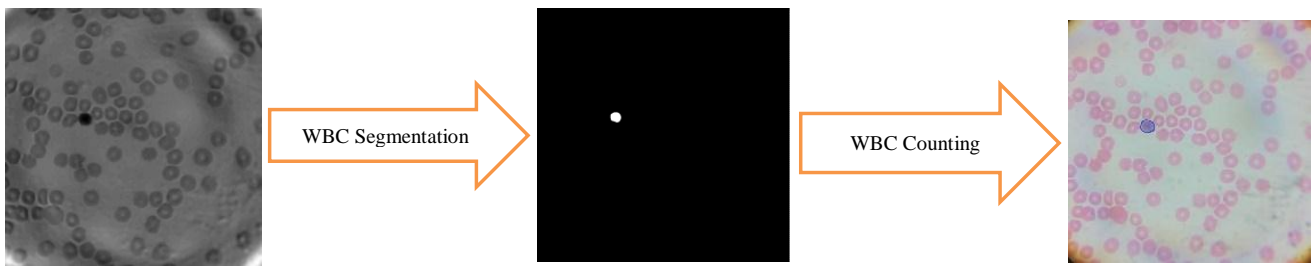

Figure $6 . b^{*}$ channel of the cropped image is used for WBC segmentation and counting

The $b^{*}$ channel image from $\mathrm{L}^{*} \mathrm{a} * \mathrm{~b}^{*}$ colour space is threshold with the value of 35 , and the binary image which had segregated WBC as foreground, is formed for WBC counting purpose. By using contour function, the WBC is captured on the binary image. Then, the system will draw the WBC contour line on the cropped image with blue colour only if the height and width of contour is more than 50 pixels. The system will also automatically count the number of contour drawn on original image. After that, the counted number is saved in global variable for further data.

In RBC segmentation and counting, the RBC in $\mathrm{a}^{*}$ channel image is segmented using binary threshold. Then, the RBC is counted using topological structural analysis, which using the same technique as WBC counting. Figure 7 illustrates the process of RBC segmentation and counting. 


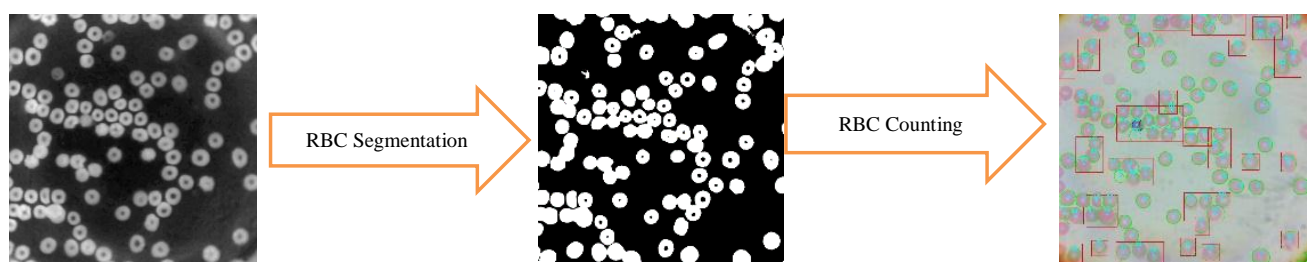

Figure 7. $\mathrm{a}^{*}$ channel of the cropped image is used for RBC segmentation and counting

The $\mathrm{a}^{*}$ channel image from $\mathrm{L}^{*} \mathrm{a} \mathrm{b}^{*}$ colour space is threshold by using Otsu method [15]. The binary image, which had segregated RBC as foreground, is formed for RBC counting purpose. By using contour function, the RBC is captured on the binary image. Then, the system will draw the RBC contour line on the cropped image with green colour only if the height and width of contour is more than 70 pixels and less than 150. The system will also automatically count the number of contour drawn on original image.

Besides, there are some clump regions of $\mathrm{RBC}$, which the height or width of the contour detected is more than 150 pixels. In order to count the RBC in the clump region, the clump region is initially cropped from the image. Next, the cropped image is undergoing Hough Circle Transform (HCT) [16]to identify the circles that found on the clump region [17]. To obtain optimum circle number, there are four parameters that require to be controlled which are accumulator threshold value, minimum distance that must be exist between two circles, minimum radius and maximum radius of the circle. In this project, such parameters are tuned to be 5.5, 75, 50 and 90 respectively. Then, the number of detected circles will also counted as RBC. After that, the counted number of RBC is saved in global variable for data management.

\subsubsection{User Interface (UI) Design}

In this phase, the UI is designed for user to insert the data and view the results. There are few UIs that had been designed, which are Sample List Activity, View List Activity and Image View Activity.

In Sample List Activity, the list of all created samples is shown. Figure 8 illustrates the UIs of Sample List Activity, View List Activity and Image View Activity.



(a)

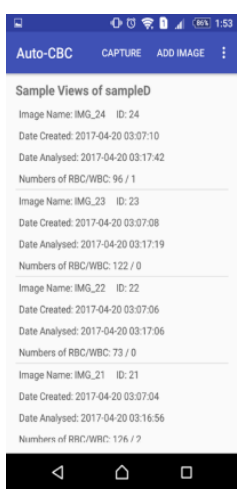

(b)



(c)

Figure 8. (a) Sample List Activity, (b) View List Activity and (c) Image View Activity

In Sample List Activity which shows in Figure 8(a), there is a list view, which contains all the samples information from the database, and an edit text field, which allow users to search the sample if there is many samples shown in the list view. Users are also allowed to press the desired sample in the sample list for obtaining the view list in the View List Activity.

Besides, there is an "Add new sample" button, which allow users to key in the sample name for adding sample into the database, and a "Reset Data" in the menu button, which allow users to clear all the data in the database including the images that stored in internal storage.

In View List Activity which shows in Figure 8(b), there is a list view, which contain all the images information from the database, and allow user to view the result of the analysis if the images is analysed. Furthermore, there is a "Capture" button, which allow users to snap a picture using camera application and 
save into internal storage, and an "Add Image" button, which allow users to copy the image from the existing storage to the internal directory for blood cell analysis purpose.

In Image View Activity which shows in Figure 8(c), the images before and after analysis are shown in the UI. Besides, the number of RBC count, WBC Count, time elapsed for the analysis time taken, date created and date analysed are shown in the UI to allow users to view the results in the Image View Activity.

\subsubsection{Image Storage and Database Management}

In this phase, the data, such as the images and other crucial information are saved at specific storage for further reference and development purposes. There are two types of data are required to be stored, which are image type and information type. Figure 9(a)

For image type data, all the images are saved in JPEG format and follow the directory as shown in

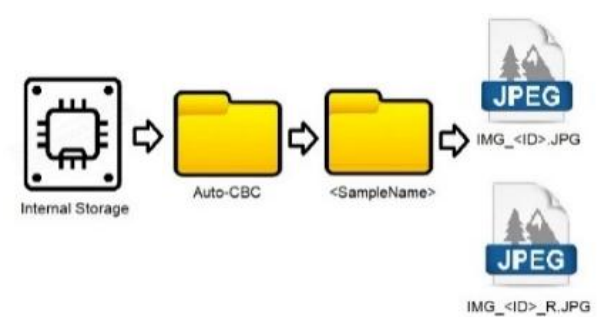

(a)


(b)

Figure 9. (a) Image files are save in the directory according to the sample name, (b) The tables with column information in SQLite database

The images type files have two classification, one is original image captured from the camera or copied from the existing storage, named as $\mathrm{IMG}_{-}<\mathrm{ID}>$.JPG ; another is the processed image after the number of RBC and WBC are counted, named as IMG_<ID>_R.JPG. There are saved in folder that according to their own parent directory, which the name of the directory is based on the sample name.

Furthermore, information type data are stored in SQLite, which is internal database that found in Android Operating System. Figure 9(b) illustrates the data arrangement in SQLite for this project.

For SQLite database, there are two tables that created in this project, which are named as "Sample" and "View". These two tables are responsible to save all the information such as the sample name, the image view name, the result of RBC and WBC count, time elapsed for the analysis time taken, date created and date modified. Hence, all of these data are able display in the UI for reference use.

\section{RESULTS AND ANALYSIS}

In this section, the performance of the system was evaluated based on the 15 images, where these images are captured from the educational type blood smear provided by the supplier. For the former assessment, the quantitative measurement is performed using confusion matrices, which are True Positive (TP), True Negative (TN), False Positive (FP) and False Negative (FN) parameters. Eventually the accuracy, precision and recall are calculated using the Equation (1). Accuracy provides evaluation regarding how well the overall system performance with respect to the ground truth data. Precision shows information about how many of detected WBC are correctly whereas recall gives how many WBC is correctly counted from the whole image, and these three parameters will be analysed on RBC.

$$
\begin{aligned}
& \text { Accuracy }=\frac{T P+T N}{T P+T N+F P+F N} ; \\
& \text { precision }=\frac{T P}{T P+F P} ; \\
& \text { recall }=\frac{T P}{T P+F N}
\end{aligned}
$$


Table 1 shows the WBC counting result analysis, and it illustrates that the average accuracy, precision and recall for the system achieve $100 \%$ in counting the WBC from blood smeared images. It shows that the system is perfect in detecting the WBC with no errors. This is because it is rare to find WBC on the educational purpose blood smear, causing each captured image only obtain 1 unit of WBC. Hence, WBC is able to be counted perfectly from the blood smeared images.

Table 2 summarises the RBC counting result, and it illustrates that the average accuracy, precision and recall for the analysis system achieve $92.93 \%, 95.19 \%$ and $97.42 \%$ respectively, in counting the RBC from blood smeared images. It shows that the system is capable and well performed to detect the RBC with few errors. There are few errors found which are miscount due to the Hough Circle Transform unable to detect the RBC in exact shape. Figure 10(a) shows example of RBC is missed from counting and miscount due to the performance of HCT.

Table 1. Result Analysis for WBC counting

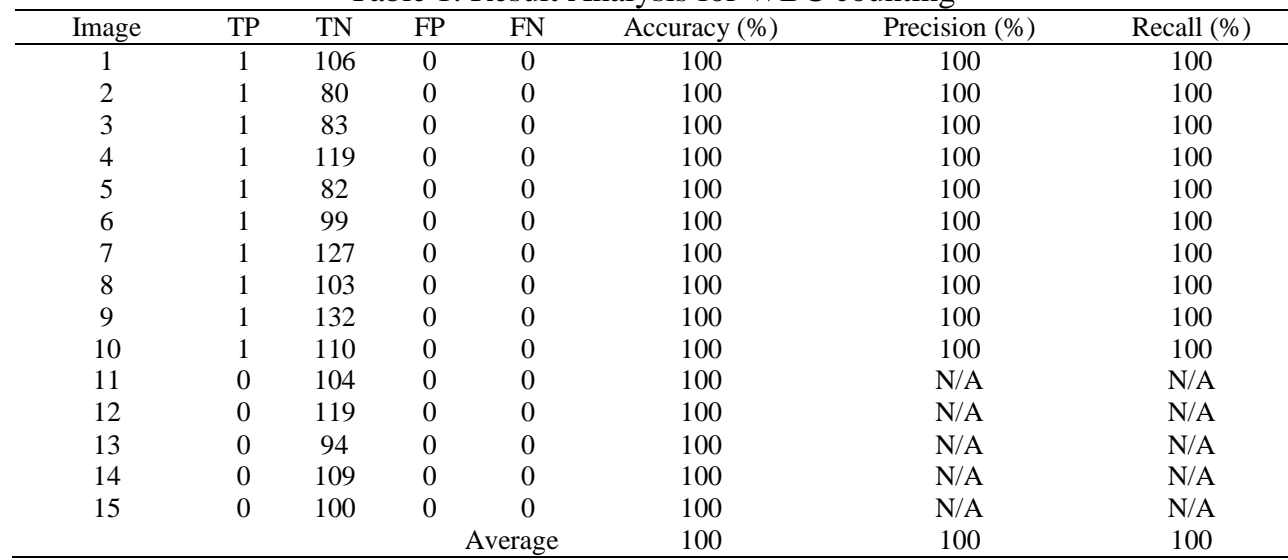

Table 2. Result Analysis for RBC counting

\begin{tabular}{cccccccc}
\hline Image & TP & TN & FP & FN & Accuracy (\%) & Precision (\%) & Recall (\%) \\
\hline 1 & 106 & 1 & 7 & 2 & 92.24 & 93.81 & 98.15 \\
2 & 80 & 1 & 0 & 4 & 95.29 & 100.00 & 95.24 \\
3 & 83 & 1 & 16 & 6 & 79.25 & 83.84 & 93.26 \\
4 & 119 & 1 & 6 & 0 & 95.24 & 95.20 & 100.00 \\
5 & 82 & 1 & 7 & 2 & 90.22 & 92.13 & 97.62 \\
6 & 99 & 1 & 3 & 2 & 95.24 & 97.06 & 98.02 \\
7 & 127 & 1 & 3 & 5 & 94.12 & 97.69 & 96.21 \\
8 & 103 & 1 & 3 & 7 & 91.23 & 97.17 & 93.64 \\
9 & 132 & 1 & 2 & 1 & 97.79 & 98.51 & 99.25 \\
10 & 110 & 1 & 5 & 2 & 94.07 & 95.65 & 98.21 \\
11 & 104 & 0 & 8 & 2 & 91.23 & 92.86 & 98.11 \\
12 & 119 & 0 & 8 & 3 & 91.54 & 93.70 & 97.54 \\
13 & 94 & 0 & 5 & 3 & 92.16 & 94.95 & 96.91 \\
14 & 109 & 0 & 2 & 1 & 97.32 & 97.20 & 99.09 \\
15 & 100 & 0 & 3 & 0 & 97.09 & 95.19 & 100.00 \\
\end{tabular}

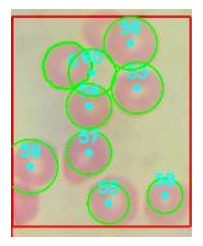

(a)

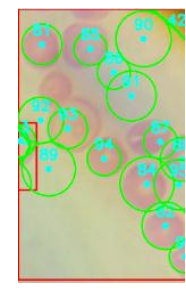

(b)

Figure 10. (a) Two RBC are missed and one contour is miscounted in the image, (b) Illumination effect (purple colour) is captured and counted as RBC with the circle number of 85,86 , and 91 
Besides, the time consumed while processing the image will be another factor of the performance in this analysis system. In order to record down the time elapsed for each image to process, the timer function is added into the application. Hence, all the time recorded are summarised in Table 3.

Table 3. Time Elapsed for processing the image

\begin{tabular}{cccc}
\hline Image & Time Record $(\mathrm{s})$ & Image & Time Record $(\mathrm{s})$ \\
\hline 1 & 3.387 & 8 & 2.356 \\
2 & 2.752 & 9 & 3.863 \\
3 & 14.171 & 10 & 3.448 \\
4 & 4.184 & 11 & 2.982 \\
5 & 2.284 & 12 & 4.990 \\
6 & 2.797 & 13 & 2.428 \\
7 & 3.210 & 14 & 2.785 \\
& & 15 & 3.616 \\
& & Average & 3.950 \\
\hline
\end{tabular}

In general, the time taken for counting nearly to $100 \mathrm{RBC}$ and $\mathrm{WBC}$ in an image is average 4 seconds, which the processing speed is consider fast, and yet there are some of the images are taking longer time, which is over than 10 seconds, to complete the processing and obtain the number of WBC and RBC.

\section{DISCUSSION}

In this section, the overall performance of developed system is discussed. In general, this system brings a bunch of advantages including high portability and high mobility, high accuracy, cheaper, less time consuming and capable to avoid human error. The developed system is very convenient, due to it is mobile based, which can bring along while travelling long distance.

Besides, this system performed high accuracy in counting RBC and WBC, with sample images tested, which makes the system more reliable, so the system is capable to avoid human error. Furthermore, the developed system has less time consuming in process. With average of 4 seconds, the system is capable to process and count the RBC and WBC automatically with high accuracy. The most important is that the cost of this developed system is lower compared to the bulky blood cell counter machine, which brings benefits to medical field, especially in developing countries.

Besides, there are few precautions being discussed, and required users to take note while implementing this analysis system. During image acquisition, the image must be cleared and the colour of the image must be high contrast in order to achieve optimum result. It is happen in the sample image 3 , which consume a longer time (14.171 seconds) to identify the blood cells. This is due to the illumination effect of the microscope, which shows in the Figure 10(b), on the contour circle 85, 86 and 91.

In order to avoid this circumstance, the smartphone camera must be firmly attached to the eyepiece magnification lens for reducing the illumination effect.

\section{CONCLUSION}

In this paper, a mobile based automated complete blood count analysis system that capable to count the number of RBC and WBC from blood smeared images is proposed. The system consists combination of three major works, which are image segmentation and counting algorithm design, user interface design, and image storage and database arrangement. Each of these works gave performance during task completion with an acceptable tolerance. The accuracy of counting RBC is $92.93 \%$ whereas WBC have a counting of $100 \%$ accuracy. Overall, this system is accurate which able to capture blood smeared image with a smartphone and counted automatically with a less time consuming of 4 seconds in average.

This system brings a lot of benefits especially for research purpose in developing country. This is because it is mobile based which is non bulky compared to blood cell counter machine, and it can save cost and time as compared to conventional method. Besides, this system can avoid human error due to pathologists do not need to count the blood cells manually. This system is low cost and high benefit which provide a convenient way to count the blood cells automatically.

Further development can be applied to make this system even more convenient such as the implementation of Internet of Things (IoT). The improvement of RBC counting can be further increase and additional function of platelet count can be develop in the future. With the growing technology of the smartphone operating system, further innovation can be done to follow the trend. This technology can be sustained developing to follow the technology trend and avoid it from being outdated. 


\section{ACKNOWLEDGEMENTS}

The authors would like to thank to Ministry of Education (MOE) and Universiti Tun Hussein onn Malaysia (UTHM) for supporting this research under Fundamental Research Grant Scheme (Vot. no. 1582). Besides, the authors would like to thank to Vitrox Corporation Berhad for providing useful information and greatly assisted throughout the research.

\section{REFERENCES}

[1] H. Tulsani, R. Gupta, and R. Kapoor, “An Improved Methodology for Blood Cell Counting,” Impact-2013, no. 1, pp. 88-92, 2013.

[2] Y. Z. Engin, K. Turhan, and A. Örem, "Prediction of Some Complete Blood Count Parameters' Values Using Boosted Regression Tree,” INISTA 2012 - Int. Symp. Innov. Intell. Syst. Appl., 2012.

[3] R. Tomari, W. Nurshzwani, W. Zakaria, and R. Ngadengon, "An Emprical Framework For Automatic Red Blood Cell Morphology Identificationi and Counting,” vol. 10, no. 19, pp. 8894-8901, 2015.

[4] A. Osei-Bimpong, R. Mclean, E. Bhonda, and S. M. Lewis, "The Use of The White Cell Count and Haemoglobin in Combination As An Effective Screen to Predict the Normality of The Full Blood Count," Int. J. Lab. Hematol., vol. 34, no. 1, pp. 91-97, 2012.

[5] S. Khan, A. Khan, and A. Naseem, "An Accurate and Cost Effective Approach to Blood Cell Count," Int. J. Comput. Appl., vol. 50, no. 1, pp. 18-24, 2012.

[6] L. E. H. Reyes, L. X. B. Rozo, and F. A. R. Morales, “Automatic Leukocyte Image Segmentation: A review,” 2015 20th Symp. Signal Process. Images Comput. Vision, STSIVA 2015 - Conf. Proc., pp. 1-9, 2015.

[7] Y. Chen, J. Guo, H. Muhammad, Y. Kang, and S. K. Ary, "CMOS-compatible Silicon-nanowire-based Coulter Counter for Cell Enumeration,” IEEE Trans. Biomed. Eng., vol. 63, no. 2, pp. 311-315, 2016.

[8] D. S. Hirschorn, A. F. Choudhri, G. Shih, and W. Kim, "Use of Mobile Devices for Medical Imaging," J. Am. Coll. Radiol., vol. 11, no. 12, pp. 1277-1285, 2014.

[9] C. L. Ventola, "Mobile Devices and Apps for Health Care Professionals: Uses and Benefits.," P T, vol. 39, no. 5, pp. 356-64, May 2014.

[10] K. Sailunaz, M. Alhussein, M. Shahiduzzaman, F. Anowar, and K. A. Al Mamun, "CMED: Cloud Based Medical System Framework for Rural Health Monitoring in Developing Countries," Comput. Electr. Eng., vol. 0, pp. 1-13, 2016.

[11] K. Chu, Z. J. Smith, and S. Wachsmann-Hogiu, "Development of Inexpensive Blood Imaging Systems: Where Are We Now?," Expert Rev. Med. Devices, vol. 12, no. 5, pp. 613-627, 2015.

[12] K. Roy, R. Dey, D. Bhattacharjee, M. Nasipuri, and P. Ghosh, "A Smart Phone Based App for Automated Segmentation and Counting of Platelets,” 2016 3rd Int. Conf. Recent Adv. Inf. Technol., pp. 434-438, 2016.

[13] D. C. Huang and K. D. Hung, "Leukocyte Nucleus Segmentation and Recognition in Color Blood-smear Images," 2012 IEEE I2MTC - Int. Instrum. Meas. Technol. Conf. Proc., pp. 171-176, 2012.

[14] Satoshi Suzuki, "Topological Structural Analysis of Digitized Binary Images by Border Following," Comput. Vision, Graph. Image Process., vol. 30, no. 1, pp. 32-46, 1985.

[15] N. Otsu, "A Threshold Selection Method from Gray-Level Histograms," IEEE Trans. Syst. Man. Cybern., vol. 9 , no. 1 , pp. 62-66, 1979.

[16] R. Tomari, W. Nurshzwani, W. Zakaria, R. Ngadengon, M. Helmy, and A. Wahab, "Red Blood Cell Counting Analysis By Considering An Overlapping Constraint," vol. 10, no. 3, pp. 1413-1420, 2015.

[17] B. Venkatalakshmi and K. Thilagavathi, "Automatic Red Blood Cell Counting Using Hough Transform," Inf. Commun. Technol. (ICT), 2013 IEEE Conf., no. Ict, pp. 267-271, 2013.

\section{BIOGRAPHIES OF AUTHORS}

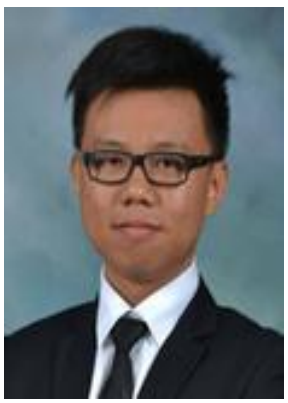

Cham Ying Kit was born in Perak, Malaysia, in 1993. He received Malaysian High School Certificate (STPM) from SMK Sultan Ibrahim Kulai in 2013. He is currently doing a Bachelor of Electronic Engineering at Universiti Tun Hussein Onn Malaysia (UTHM). His current interests include mobile application development, machine visison, image processing, and medical robotics system. 



Razali Tomari was born in Johor, Malaysia, in 1980. He received the B.Eng degree in Mechatronics from the Universiti Teknologi Malaysia, in 2003, M.Sc in intelligent system from the Universiti Putra Malaysia, in 2006. And $\mathrm{PhD}$ degree in computer vision and robotic from the Saitama University, Japan in 2013. In 2003, he joined Faculty of Electrical Engineering, University Tun Hussein Onn as a tutor and later on become a Senior Lecturer in 2013. His current research interest includes computer vision, pattern recognition, smart wheelchair and sensing technology.

WN Wan Zakaria received B.Eng (2007) in Electronics and Mechanical Engineering from Chiba University and MSc(2008) and PhD (2012) from Newcastle University. She is currently a lecturer in Faculty of Electrical and Electronic Engineering, Universiti Tun Hussein Onn Malaysia. Her current interests include Medical Robotics System specifically on development of robot force control, Image Processing and Computer Aided Diagnosis, and development of Wearable Device. She is author and co-author of several journal papers and conference proceedings.

Nurmiza Binti Othman was born in Terengganu, Malaysia, in 1983. She received the M. Sc. (Electrical and Electronic Engineering) from Utsunomiya University, Japan in 2009 and the Ph.D (Electrical and Electronic Engineering) from Kyushu University, Japan in 2014. In 2007, she joined the Department of Electronic Engineering, Universiti Tun Hussein Onn Malaysia as a tutor. From 2014 to present, she has appointed as a lecturer with the same institution. Currently, she is also a Senior Researcher of UTHM Research Center For Applied Electromagnetics, Universiti Tun Hussein Onn Malaysia. She is a member of professional membership IEEE under Engineering in Medicine and Biology Society. Her research interests include Superconductor Engineering, Microfabrication, Nanomagnetic Materials, Magnetic Particle Imaging, and Magnetic Tomography

Syadia Nabilah was born in Kuala Lumpur, Malaysia, in 1993. She received B.Eng degree in Electronic from Universiti Tun Hussein Onn Malaysia (UTHM), in 2016. She is currently doing a MEng in Electrical at UTHM. Her ongoing research is in Leukemia detection by applying a computer vision system for image processing purposes. Her current interests include Medical Robotics System specifically on Image Processing and Computer Aided System.

Nicholas Tan Chun Sheng was born in Penang, Malaysia in 1994. He received Malaysian High School Certificate (Physics) from Heng Ee High School. He is currently doing a Bachelor of Electronic Engineering at Universiti Tun Hussein Onn Malaysia (UTHM). His current interests include integrated circuit design, device simulation (TCAD) and user interface and experience (UI and UX) design.

Jacqueline Ang Jie Yi was born in Kuala Lumpur, Malaysia, in 1994. She received Malaysian Higher School Certificate (STPM) from SMK Batu 8, Jalan Puchong in 2013. She is currently doing a Bachelor Degree of Electronic Engineering at Universiti Tun Hussein Onn Malaysia (UTHM). Her current interests include programming and electronic circuit design. 